\title{
Extension of the Isobolographic Approach to Interactions Studies between More than Two Drugs: Illustration with the Convulsant Interaction between Pefloxacin, Norfloxacin, and Theophylline in Rats
}

\author{
CÉLINE BROCHOT, ${ }^{1}$ SANDRINE MARCHAND, ${ }^{2}$ WILLIAM COUET, ${ }^{2}$ ANDREW GELMAN, ${ }^{3}$ FRÉDÉRIC Y. BOIS ${ }^{1}$ \\ ${ }^{1}$ Institut National de l'Environnement Industriel et des Risques, Unité de Toxicologie Expérimentale, Parc Alata, \\ BP2, 60550 Verneuil-En-Halatte, France \\ ${ }^{2}$ EE Médicaments Anti-infectieux et BHE, Pôle Biologie Santé, Faculté de Médecine \& Pharmacie, \\ 40 Avenue du Recteur Pineau, 86022 Poitiers Cedex, France \\ ${ }^{3}$ Department of Statistics, Columbia University, New York, New York 10027, USA
}

Received 2 April 2003; revised 21 August 2003; accepted 27 August 2003

\begin{abstract}
This work proposes a model to characterize the additivity or the nonadditivity of combinations of more than two agents. Using a Bayesian framework, we modeled the variability between experimental subjects, the errors that occurred during data collection, and the relationship between effects and concentrations of agents at the effect site. The model was used to characterize the additivity (or non-additivity) of norfloxacin, pefloxacin, and theophylline in causing maximal seizures in male Sprague Dawley rats. Animals received the drugs separately or in various combinations. Drug infusion was stopped at the onset of maximal seizures, and cerebrospinal fluid samples were collected for determination of drug concentration by high-performance liquid chromatography. The model was fitted to concentration data using Markov Chain Monte Carlo techniques. Results showed that induction of seizures by mixtures of theophylline and pefloxacin were additive. Seizure induction by mixtures of norfloxacin and pefloxacin or norfloxacin and theophylline were not additive and, given the model, these drugs interacted negatively. There was no triple interaction effect between the drugs. This study demonstrates the ease with which mixtures of more than two drugs can be analyzed with the proposed model. (c) 2004 Wiley-Liss, Inc. and the American Pharmacists Association J Pharm Sci 93:553-562, 2004

Keywords: drug interaction; pharmacodynamics; Monte Carlo; simulations; mathematical model; blood cerebrospinal fluid barrier
\end{abstract}

\section{INTRODUCTION}

Because patients are often treated with several drugs in combination, it is of primary importance to characterize potential drug-drug interactions, which may occur at a pharmacokinetic (PK) as well as a pharmacodynamic (PD) levels. PK

Correspondence to: Frédéric Y. Bois (Telephone: 3334455 65 96; Fax: 333445566 05; E-mail: frederic-bois@ineris.fr)

Journal of Pharmaceutical Sciences, Vol. 93, 553-562 (2004)

(c) 2004 Wiley-Liss, Inc. and the American Pharmacists Association interaction studies are relatively easy to set up and therefore frequently conducted. By contrast, PD interactions in vivo are more difficult to assess, in particular because it may be difficult to characterize PD outcomes (particularly when surrogate markers of effect have to be used) and to distinguish between the PK and PD contributions to the observed effect.

The convulsant activity of several drugs, including theophylline and the pefloxacin and norfloxacin fluoroquinolones antibiotics, can be assessed by measurement of their concentrations in the 
cerebrospinal fluid (CSF), which is part of the biophase, at the onset of maximal seizures. ${ }^{1,2}$ Such an approach enables the isolation of the PD of the convulsant effect of the drug from its PK characteristics [i.e., ability to reach its pharmacological receptors at the central nervous system (CNS)]. This method has also been used to characterize PD interactions among these various compounds using a newly developed isobolographic approach. ${ }^{3-5}$ However, a major limitation of the latter approach is that it is restricted to the combination of two drugs. The objective of the present study was therefore to develop a new approach that would extend the isobolographic approach to combinations of more than two compounds.

\section{EXPERIMENTAL}

Part of the data used for this analysis were obtained in rats treated with pefloxacin, norfloxacin, and theophylline alone or in binary mixtures, and have previously been published. ${ }^{3-5} \mathrm{~A}$ complementary data set corresponding to the administration of ternary mixtures of these three compounds has been generated as follows.

\section{Solutions for Administration}

Exposure to theophylline (Assistance Publique des Hôpitaux de Paris, Paris, France) was accomplished by intramuscular (im) or intravenous (iv) administration of a $25 \mathrm{mg} / \mathrm{mL}$ solution of aminophylline (corresponding to theophylline at $19.7 \mathrm{mg} / \mathrm{mL}$ or $109 \mathrm{mM}$ ). Pefloxacin was administered as a commercially available solution of pefloxacin methane sulfonate (Bellon Laboratories, Neuilly Sur Seine, France) at a concentration of $80 \mathrm{mg} / \mathrm{mL}$ or $240 \mathrm{mM}$. Norfloxacin (Sigma, Saint-Quentin Fallavier, France) was infused as a $76.6 \mathrm{mg} / \mathrm{mL}$ solution of norfloxacin hydrochloride (corresponding to $240 \mathrm{mM}$ ) dissolved in $5 \%$ glucose at $\mathrm{pH} 5.5$. $^{1,5,6}$

\section{Animals}

This work was done in accordance with the Principles of Laboratory Animal Care (NIH Publication \#85-23, revised 1985), and the local ethics committee approved the study protocol. Male Sprague Dawley rats $(n=23)$ from Dépré Breeding Laboratories (Saint-Doulchard, France), with body weights at arrival ranging between 220 and
$240 \mathrm{~g}$ and a mean \pm standard deviation (SD) body weight at the time of dosing of $245 \pm 10 \mathrm{~g}$, were housed in the Animal Breeding Facilities of the Laboratory (authorization no. 0028). The animals were placed in wire cages in a 12-h light/dark cycle for 5 days before the beginning of the experiment to accustom them to their new environment. During this period, they had free access to food (U.A.R. AO4; UAR Laboratories, Villemoisson sur Orge, France) and water.

\section{Surgery}

Surgery was carried out as previously described. ${ }^{3-5}$ Because of the physicochemical incompatibilities between the theophylline and the fluoroquinolones solutions, two polyurethane catheters $(0.51 \mathrm{~mm}$ inside diameter, $0.71 \mathrm{~mm}$ outside diameter; Plastimed Laboratories, France) were implanted in the left jugular vein of the animals.

\section{Drug Administration}

The day after surgery, the jugular vein cannula was connected to a two-way motor-driven syringe pump (SE400B, Vial Medical, St Etienne de St Geoirs, France) equipped with two syringes, one containing an equimolar mixed solution of pefloxacin and norfloxacin and the other containing the solution of theophylline. Based on previous experiments, ${ }^{3-5}$ it was estimated that by adjusting the flow rates of the two syringes to $2 \mathrm{~mL} / \mathrm{h}$, each of the three compounds would contribute more or less equally to the observed effect. Therefore, 10 rats were treated accordingly. The flow rates were adjusted differently in the 13 remaining animals to change the relative contribution of each compound to the effect. In all cases, the total flow rate was kept equal to $4 \mathrm{~mL} / \mathrm{h}$. During infusion, rats were kept under a heating lamp to maintain body temperature. The infusion was stopped when the animals exhibited maximal seizures. ${ }^{3-5}$ Onset of maximal seizures was usually evidenced by tonic flexion of the forelimbs and tonic extension of the hind limbs. Drug administration was conducted between 2:00 p.m. and 7:00 p.m.

\section{Sample Collection}

Immediately after exhibiting maximal seizures, the rats were anesthetized with an im injection of ketamine (KETALAR ${ }^{\mathbb{R}}, 50 \mathrm{mg} / \mathrm{mL}$; Parke Davis 
Laboratories, Courbevoie, France) plus xylazine hydrochloride (ROMPUN ${ }^{\circledR}$; Bayer Laboratories, Luteaux, France), unless they had died following seizures. CSF was collected following the end of infusion, as previously described. ${ }^{1,3-5}$

\section{Drug Analysis}

The three compounds were simultaneously assayed in CSF samples by high-performance liquid chromatography (HPLC) with ultraviolet (UV) detection at $280 \mathrm{~nm}$ as previously described. $^{3-5}$ Quality control data (not shown) with various known concentration probes in the CSF indicated that the coefficients of variation of the assay technique were at most $8.8,9.7$, and $9 \%$ for theophylline, norfloxacin, and pefloxacin, respectively. Corresponding biases were at most 9, -6 , and $12.5 \%$ respectively. The limits of quantification were much below measured values for any of these compounds.

\section{Data Modeling}

For simplicity, the model is first presented for two drugs. Because for the drugs studied the onset of maximal seizures is related to concentration in the CSF (part of the biophase) ${ }^{1,2}$ rather than to plasma concentration or dose, this PD model starts with CSF concentrations as input.

An important aspect of our model is the integration of the uncertainty of the measurements in the CSF, which are made only with finite precision. We note by $x_{i}$ and $u_{i}$, respectively, the measured and the actual concentration of the drug $i$ at the site of interest. We suppose that the measured concentration, $x_{i}$, is log normally distributed with geometric mean, $u_{i}$, and geometric standard deviation, $\sigma_{i}$. So the measurement error model is given by:

$$
\log x_{i} \sim N\left(\log u_{i},\left(\log \sigma_{1}\right)^{2}\right)
$$

In eq. 1 , the $\sim$ symbol means "statistically distributed as...". No mechanistic information is available in our data on the relationship between drug concentration and response. However, a threshold model for seizures is reasonable. We therefore postulate that an underlying variable, $y$, related to concentration, crosses a threshold, $y_{\mathrm{E}}$, when the target effect is recorded. The value of $y_{\mathrm{E}}$ itself remains unobserved. We suppose that the relationship between $y$ and the concentrations $u_{i}$ is given by a standard polynomial function. ${ }^{7}$ For a mixture of two drugs, this model is:

$$
y=\theta_{0}+\theta_{1} u_{1}+\theta_{2} u_{2}+\theta_{12} u_{1} u_{2}
$$

where $\theta_{0}$ is the parameter associated with the effect of the control concentration (usually concentration zero), $\theta_{i}$ is the parameter associated with the effect of drug $i$, and $\theta_{12}$ is the parameter describing the sign and strength of interactions between the two agents. ${ }^{8}$ If the concentrationresponse relationship were to be known, it would be very simple to change eq. 2 accordingly.

To account for interindividual differences in susceptibility to convulsion, we assume that each subject $j$ has a different threshold, $y_{\mathrm{E}, j}$, and embed the model in the framework of a standard population model. ${ }^{9}$ If the values of the $\theta$ parameters are subject independent, the polynomial model, at the threshold level of effect $y_{\mathrm{E}, j}$, can be written as

$$
y_{\mathrm{E}, j}=\theta_{0}+\theta_{1} u_{1, j}+\theta_{2} u_{2, j}+\theta_{12} u_{1, j} u_{2, j}
$$

and the corresponding isobol is

$$
u_{2, j}=\frac{y_{\mathrm{E}, j}-\theta_{0}-\theta_{1} u_{1, j}}{\theta_{2}+\theta_{12} u_{1, j}}
$$

Parameters $y_{\mathrm{E}, j}$ and $\theta_{0}$ are not separately identifiable, and each parameter can be divided by $\theta_{1}$. So the simplest reparametrized isobol is

$$
u_{2, j}=\frac{y_{\mathrm{E}, j}^{\prime}-u_{1, j}}{\theta_{2}^{\prime}+\theta_{12}^{\prime} u_{1, j}}
$$

where

$$
y_{\mathrm{E}, j}^{\prime}=\frac{y_{\mathrm{E}, j}-\theta_{0}}{\theta_{1}}, \quad \theta_{2}^{\prime}=\frac{\theta_{2}}{\theta_{1}}, \quad \theta_{12}^{\prime}=\frac{\theta_{12}}{\theta_{1}}
$$

At the population level, individual reparameterized thresholds, $y_{\mathrm{E}, j}^{\prime}$, are supposed to be log normally distributed with mean $M_{y}^{\prime}$ and standard deviation $\sigma_{y}^{\prime}$ :

$$
\log y_{\mathrm{E}, j}^{\prime} \sim N\left(\log M_{y}^{\prime},\left(\log \sigma_{y}^{\prime}\right)^{2}\right)
$$

This population model is related to the probit model $;{ }^{10}$ that is, effects are observed when the response goes over a log normally distributed threshold value. A graphical representation ${ }^{11}$ of the model is shown Figure 1. The constants (for example, the data) on the figure are placed in rectangles and random variables are boxed in circles. The line between parameters $u_{1}$ and $u_{2}$ marks a deterministic relation between these parameters. An arrow between two nodes indicates a direct statistical dependence between the 


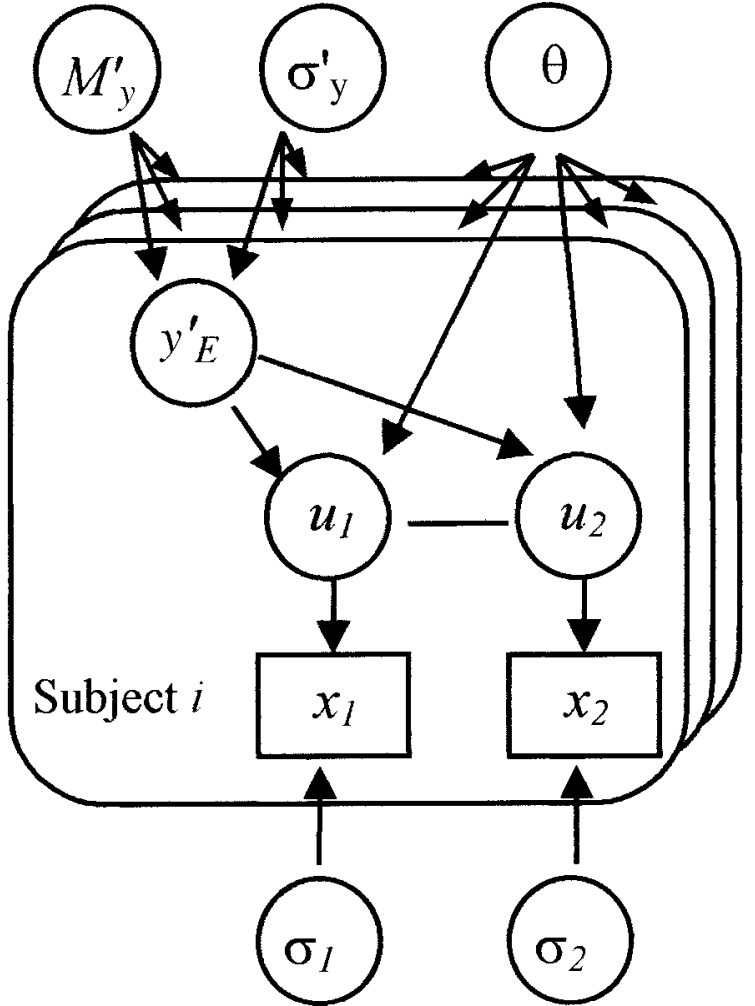

Figure 1. Graph of the hierarchical statistical model describing dependencies between variables. Symbols are: $M_{y}^{\prime}$, geometric mean of thresholds; $\sigma_{y}^{\prime}$, geometric standard deviation of thresholds; $\theta$, unknown parameters corresponding to the concentration-response relationship; $y_{\mathrm{E}}^{\prime}$, thresholds; $u_{1}$ and $u_{2}$, real concentrations of agents A and B, respectively; $x_{1}$ and $x_{2}$, measured concentrations of the two drugs; and $\sigma_{1}$ and $\sigma_{2}$, geometric standard deviations corresponding to measurement errors. Variables of unknown value are denoted as circles, and constant values are denoted as rectangles. The line between $u_{1}$ and $u_{2}$ marks a deterministic relation. The stack of "cards" (behind the front one) represent the corresponding model for the other subjects.

variables of those nodes. For a two-drug mixture, the set of parameters to estimate $\Theta$ is,

$$
\Theta=\left\{\theta_{2}^{\prime}, \theta_{12}^{\prime}, \overrightarrow{y_{\mathrm{E}}^{\prime}}, M_{y}^{\prime}, \sigma_{y}^{\prime}, \overrightarrow{u_{1}}, \overrightarrow{u_{2}}, \sigma_{1}, \sigma_{2}\right\}
$$

For three interacting drugs, the measurement error model and the population model are the same (eqs. 1 and 6), and eq. 5 has to be replaced by

$$
u_{1, j}=\frac{y_{\mathrm{E}, j}^{\prime}-\theta_{2}^{\prime} u_{2, j}-\theta_{3}^{\prime} u_{3, j}-\theta_{23}^{\prime} u_{2, j} u_{3, j}}{1+\theta_{12}^{\prime} u_{2, j}+\theta_{13}^{\prime} u_{3, j}+\theta_{123}^{\prime} u_{2, j} u_{3, j}}
$$

(see Appendix A for details). In this case, the set of parameters to estimate $\Theta$ is

$$
\begin{aligned}
\Theta= & \left\{\theta_{2}^{\prime}, \theta_{3}^{\prime}, \theta_{12}^{\prime}, \theta_{13}^{\prime}, \theta_{23}^{\prime}, \theta_{123}^{\prime}, \overrightarrow{y_{\mathrm{E}}^{\prime}}, M_{y}^{\prime}, \sigma_{y}^{\prime}, \overrightarrow{u_{1}}, \overrightarrow{u_{2}},\right. \\
& \left.\overrightarrow{u_{3}}, \sigma_{1}, \sigma_{2}, \sigma_{3}\right\}
\end{aligned}
$$

\section{Bayesian Analysis}

In a Bayesian framework, ${ }^{12,13}$ all unknowns (the set of parameters, $\Theta$ ) are considered as random variables. Before analyzing the experimental data, a prior probability distribution, $P_{\Theta}$, is proposed for each unknown parameter. This probability distribution can be interpreted in terms of degrees of beliefs about the possible values of $\Theta$ and should reflect the current knowledge on $\Theta$. Knowledge includes, for example, in vitro data or information found in literature. Precise or informative distributions can be set for parameters whose possible values are well known. When little information is available, flat, noninformative distributions can be used.

The set of prior distributions is then updated using the new experimental data, $x$, to yield a posterior probability distribution. Updating is performed using Bayes' theorem, which indicates that the posterior probability distribution, $P(\Theta \mid x)$, is proportional to the product of all the prior distributions, $P_{\Theta}$, by the data likelihood, $P(x \mid \Theta)$ :

$$
P(\Theta \mid x) \propto P_{\Theta} \times P(x \mid \Theta)
$$

The form of the likelihood depends on the error model and on the deterministic model chosen. The posterior probability distribution is a representation of the knowledge on the parameters, including the information contained in the data.

Any statistical summary on the parameters (e.g., mean, standard deviation, percentiles) can be extracted from their posterior distribution. However, it is often difficult to obtain an analytical expression for the joint posterior distribution, $P(\Theta \mid x)$. Fortunately, Markov Chain Monte Carlo (MCMC) methods alleviate this difficulty. ${ }^{14}$ These methods provide samples of parameters values from their posterior distribution even if an analytical expression for it cannot be obtained.

So, a prior distribution was first defined for all model parameters. The measurement error model was already defined as log normal (eq. 1) and the distribution of the $y_{\mathrm{E}, j}^{\prime}$ was also set to a log normal 
distribution (eq. 6). In eq. $5, u_{1, j}$ has to be less than $y_{\mathrm{E}, j}^{\prime}$ so a uniform distribution between 0 and $y_{\mathrm{E}, j}^{\prime}$ for each $u_{1, j}$, or $u_{2, j}$ in the case of treatment by agent $\mathrm{B}$ alone. Only prior distributions for the parameters of the concentration-response relationship ( $\theta_{2}^{\prime}$ and $\theta_{12}^{\prime}$ for a mixture with two drugs), for the mean and the SD of the thresholds $\left(M_{y}^{\prime}\right.$ and $\left.\sigma_{y}^{\prime}\right)$ and for the SD related to the measurements error model $\left(\sigma_{i}\right)$ have to be defined. Here, uniform noninformative prior distributions were used for parameters of the concentration-response relationship and for the geometric mean $M_{y}^{\prime}$ of individual thresholds. Inverse-gamma distributions were used for the threshold variance $\left(\log \sigma_{y}^{\prime}\right)^{2}$, and the measurement error variances $\left(\log \sigma_{i}\right)^{2}$. This choice is standard for variances of normal distributions. ${ }^{12,13}$ For threshold variance, the shape parameter of the inverse-gamma distribution was set to 2 , and the inverse scale parameter was set to 0.06 to obtain a coefficient of variation of $25 \%$ (of the order of the interindividual variability observed in PK studies ${ }^{9}$ ). For measurement of error variances, the shape and inverse-scale parameters were those of the posterior distribution of variance in a Normal model with known average, given noninformative priors and the independent quality assurance data available to us (not shown); ${ }^{12}$ that is, quality assurance data were used to obtain distributions for variances, which were in turn used as prior distributions in our interaction model. For norfloxacin, shape and inverse scale were set, respectively, to 57.5 and 0.32 [so the expectation of $\left(\log \sigma_{\mathrm{N}}\right)^{2}$ for norfloxacin is 1.08, which corresponds to a coefficient of variation of $8 \%$ in natural scale). For pefloxacin, these values were set to 55.5 and 0.32 , respectively, and for theophylline to 26.5 and 0.145 , respectively (these values correspond to a coefficient of variation of $8 \%$ in natural scale).

Fitting the population model to the data was performed with MCMC techniques (Metropolis sampling) using the MCSim Software. ${ }^{15}$ The model file and the data file are available from the first author upon request. Five independent Markov chains of 6.5 millions iterations each were run. For each chain, only one simulation in 50 of the last 500,000 simulations was recorded. So, a total of 50,000 vectors of parameters were recorded and used to produce the following results. For each parameter, the convergence diagnostic $\hat{R}$ was used to check convergence to a stable posterior estimate. ${ }^{13}$ If $\hat{R}$ was $<1.1$ for all parameters, approximate convergence was supposed to be achieved.

\section{RESULTS}

Concentrations of drug(s) in CSF at the onset of maximal seizures for 150 rats were used in this analysis. Norfloxacin, pefloxacin, and theophylline were administered alone to 20,15 , and 14 rats, respectively. A total of 25 rats received a mixture of norfloxacin and pefloxacin, 13 received norfloxacin and theophylline, and 40 rats received pefloxacin and theophylline. The three drugs were administered jointly to 23 rats. Data on these 23 rats have not been previously published. All the data were analyzed together with our model, and posterior simulations were obtained for all parameters. The data with 20 isobols for each binary mixture, corresponding to 20 randomly-drawn parameter vectors are shown in Figure 2. Summary statistics of the marginal posterior distribution of each parameter are given in Table 1. Using these distributions, it is possible to assess the additivity or the nonadditivity of the effect of the drugs on convulsions for each mixture.

For example, the $\theta_{\mathrm{PT}}^{\prime}$ parameter measures interaction between pefloxacin and theophylline effects on convulsions. As can be seen in Figure 2A, the isobols have no appreciable curvature. The 95\% posterior interval of $\Theta_{\mathrm{PT}}^{\prime}$ is $[-0.000022$; $0.000110]$, which includes zero $(12 \%$ of the sampled values fall below it). The curvature is therefore not statistically significant, in addition to not being biologically significant, as is clear from Figure 2A. It can be concluded that, given our model, the effect of mixtures of pefloxacin and theophylline in the studied dose range are approximately additive.

For norfloxacin and theophylline, $98.7 \%$ of the sampled values of $\theta_{\mathrm{NT}}^{\prime}$ fall below zero, and its mean is -0.000374 , denoting a significant antagonism (given the model). Some isobols are presented Figure 2B. To characterize the magnitude of this antagonism, eq. 5 can be rewritten as

$$
y_{\mathrm{E}, j}^{\prime}=u_{1, j}\left(1+\theta_{12}^{\prime} u_{2, j}\right)+\theta_{2}^{\prime} u_{2, j}
$$

This equation can be interpreted by considering that the effect of agent $\mathrm{A}$ (produced with concentration $u_{1, j}$ ) is altered by the presence of agent $\mathrm{B}$ (concentration $u_{2, j}$ ). For example, consider the couple $(40,734)$ of concentrations of norfloxacin and theophylline. For $\theta_{\mathrm{NT}}^{\prime}=-0.000374$ and at the level of effect, the concentration of norfloxacin is apparently lowered by $27.5 \%$ by the presence of theophylline. This percentage is specific to the considered concentration values. 


\section{Theophylline}

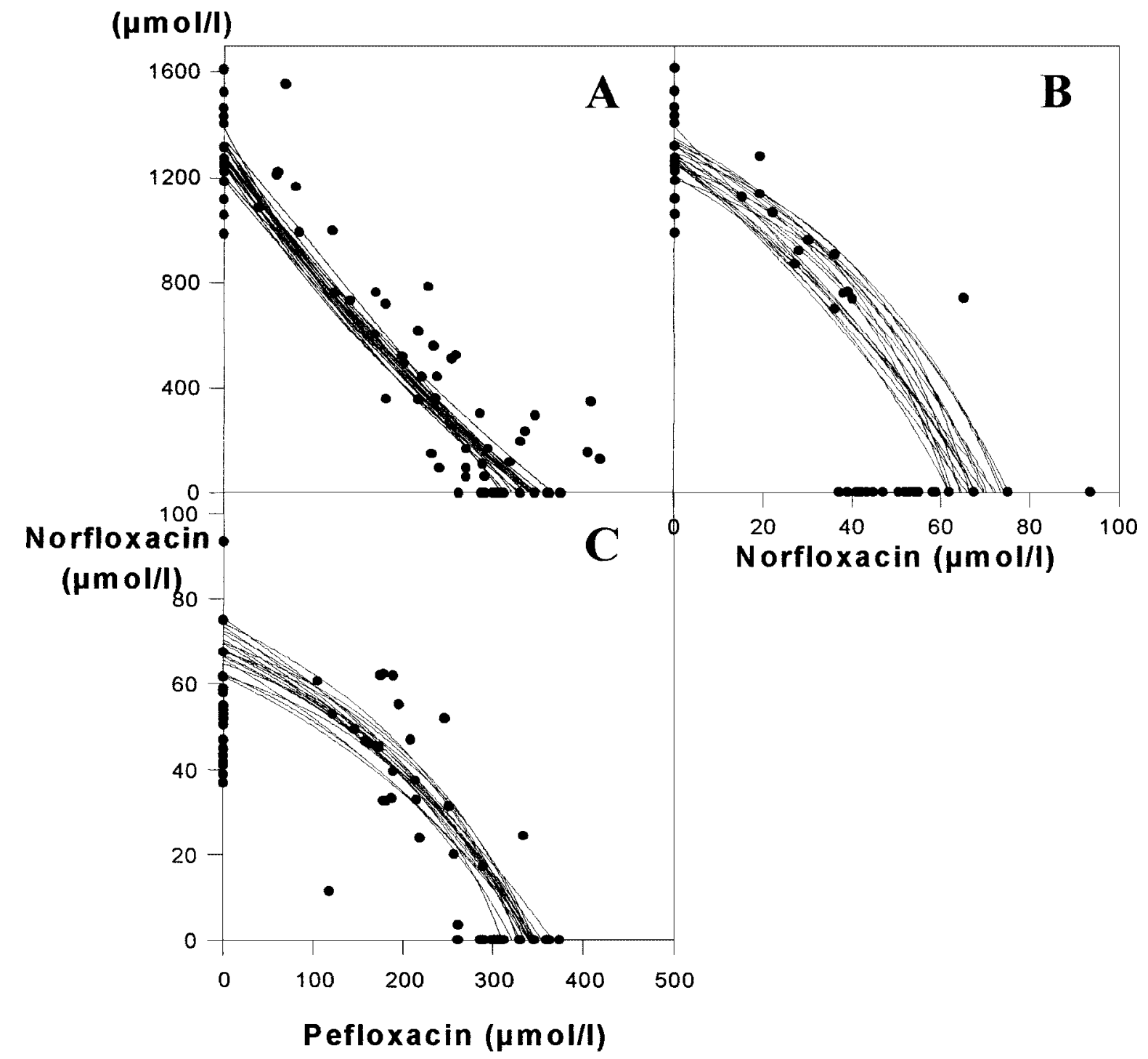

Figure 2. Pairwise interaction data (dots) between (A) pefloxacin and theophylline, (B) norfloxacin and theophylline, and (C) norfloxacin and pefloxacin, together with isobols (lines) corresponding to 20 random simulations from the posterior distribution.

For norfloxacin and pefloxacin, the curvature of the isobols displayed on Figure 2C indicates apparent antagonism again. This observation is confirmed by the posterior distribution of $\theta_{\mathrm{NP}}^{\prime}$; $99.8 \%$ of its sampled values fall below zero and its mean is -0.001148 .

The nature of the interaction between the three drugs is characterized by the parameter $\theta_{\mathrm{NPT}}^{\prime}$. Zero is included in its $95 \%$ posterior interval, so there is no extra effect of having the three drugs together beyond the two-by-two interactions already described above. Only $11 \%$ of the esti- mated values of $\theta_{\mathrm{NPT}}^{\prime}$ are positive. An estimated response surface, the parameters of which correspond to the mode of the joint posterior parameter distribution (the vector of estimated parameters with the highest likelihood), is presented in Figure 3.

\section{DISCUSSION}

A general framework and an original statistical model were proposed to assess additivity in a 
Table 1. Summary Statistics for the Marginal Posterior Distributions of the Parameters in the Study of the Additivity between Norfloxacin, Pefloxacin, and Theophylline ${ }^{a}$

\begin{tabular}{|c|c|c|c|c|c|}
\hline Parameter & Symbol & Mean & $\begin{array}{l}\text { Standard } \\
\text { Deviation }\end{array}$ & $\begin{array}{c}2.5 \text { th } \\
\text { Percentile }\end{array}$ & $\begin{array}{c}\text { 97.5th } \\
\text { Percentile }\end{array}$ \\
\hline Modified population threshold & $M_{\gamma}^{\prime}$ & 67.4 & 3.60 & 61.4 & 75.7 \\
\hline Interindividual variability & $\sigma_{y}^{\prime}$ & 1.20 & 1.09 & 1.16 & 1.25 \\
\hline Potency ratio of pefloxacin to norfloxacin & $\theta_{\mathrm{P}}^{y}$ & 0.199 & 0.0119 & 0.178 & 0.225 \\
\hline Potency ratio of theophylline to norfloxacin & $\theta_{\mathrm{T}}^{\prime}$ & 0.0529 & 0.0039 & 0.0463 & 0.0616 \\
\hline Interaction parameter (NP) & $\theta_{\mathrm{NP}}^{\prime}$ & -0.00148 & 0.000406 & -0.00218 & -0.000579 \\
\hline Interaction parameter (NT) & $\theta_{\mathrm{NT}}^{\prime}$ & -0.000374 & 0.000148 & -0.000639 & -0.000054 \\
\hline Interaction parameter (PT) & $\theta_{\mathrm{PT}}^{\prime N I}$ & 0.000037 & 0.000033 & -0.000022 & 0.000110 \\
\hline Interaction parameter (NPT) & $\theta_{\mathrm{NPT}}^{\prime}$ & $-2.24 \times 10^{-6}$ & $1.93 \times 10^{-6}$ & $-6.36 \times 10^{-6}$ & $1.32 \times 10^{-6}$ \\
\hline Measurement error (norfloxacin) & $\sigma_{\mathrm{N}}$ & 1.08 & 1.03 & 1.07 & 1.09 \\
\hline Measurement error (pefloxacin) & $\sigma_{\mathrm{P}}$ & 1.08 & 1.03 & 1.07 & 1.09 \\
\hline Measurement error (theophylline) & $\sigma_{\mathrm{T}}$ & 1.08 & 1.03 & 1.06 & 1.09 \\
\hline
\end{tabular}

${ }^{a}$ Units: $M_{y}^{\prime}$ is in $\mu \mathrm{mol} / \mathrm{L}$. The other parameters are unitless.

specific experimental scheme (direct assays). The problem in direct assays is that the framework is not a standard regression one. Concentrations of drugs and mixture of drugs that lead to the target effect are observed (and in our case, they are actually measured) with error. In addition, for a binary response, an underlying threshold to estimate has to be introduced. Further, there may be variability issues if each data point corresponds to a different animal or experimental unit. To analyze this kind of data, we developed a statistical model in a Bayesian context, reducing the analysis of direct assays data to a well-defined problem of statistical inference based on an underlying concentration-response model. The model presented in this paper belongs to the class of "indirect link PK/PD models", in which a PK model is used to predict effect site concentrations

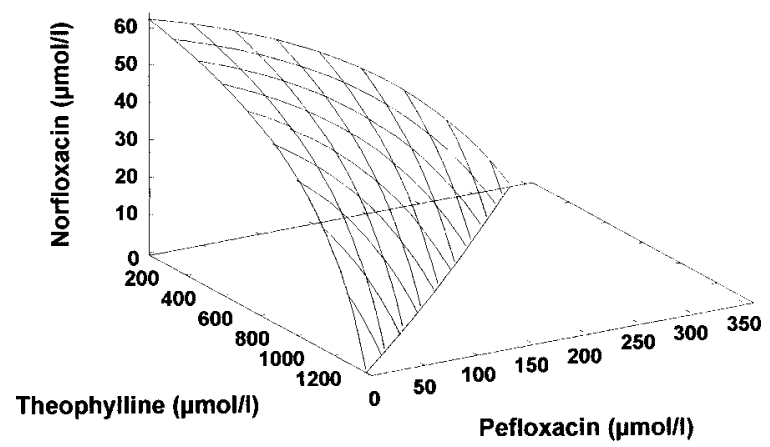

Figure 3. Convulsion threshold surface for mixtures of norfloxacin, pefloxacin, and theophylline. The parameters of the surface correspond to the mode of the joint posterior parameter distribution. of drugs and a PD model links that concentration to observed effects. ${ }^{16}$ In our case, however, a PK model was not needed to predict CSF concentrations because they were actually measured. To take into account the fact that these data were affected by measurement error, an "errors-invariables" approach ${ }^{17}$ was used (similarly to what Bennett and Wakefield suggest when decoupling $\mathrm{PK}$ and PD models ${ }^{18}$ ). Independent quality assurance data were used to estimate $a$ priori the size of measurement errors. We also defined a population model to separate measurement error from interindividual variability.

Effects of mixtures of two of the three drugs studied here (norfloxacin, pefloxacin, and theophylline) have already been published, ${ }^{3}$ and a discrepancy between those results and ours was observed in one occasion. A slight but statistically significant negative interaction was previously reported between pefloxacin and theophylline, ${ }^{3}$ whereas using our new model, we concluded simple additivity. The approach used in the initial report requires a relatively large number of data points for the drugs administered separately to estimate average threshold concentrations, but only five CSF concentrations at the onset of maximal seizures were available for theophylline and three for pefloxacin. These numbers of available concentrations were probably not enough to precisely estimate threshold concentrations. Our modeling approach does not suffer from this limitation, which is likely to explain the discrepancy. Antagonism (in this case, the mutual lowering of the convulsant effect of the drug) between pefloxacin and norfloxacin ${ }^{6}$ and between theophylline 
and norfloxacin ${ }^{5}$ were found with the initial approach and confirmed using our new model. The results obtained with the two approaches are therefore quite consistent. Yet, compared with the previous approach, ${ }^{3}$ a definite advantage of our new model, in addition to statistical improvements, is the ease with which it can describe the effects of mixtures of more than two drugs.

There is potential to improve our model further. For example, in our population model, individual thresholds were assumed to be log normally distributed (this corresponds to a log normal probit model). This choice is classical for biological parameters, which take strictly positive values, ${ }^{19}$ and it has allowed us to define an informative prior distribution for thresholds. However, other distributions could be used. Alternatively, the use of a logit model could be explored.

We have chosen to work with a flexible empirical polynomial model of interactions. This model includes, as special cases, the isobols corresponding to mixtures of drugs for which the marginal concentration-response models are linear or of $E_{\text {max }}$ type. In particular, if the $E_{\text {max }}$ model is used instead of the linear model, the isobol has the same expression as in eq. 5 (see Appendix C). The interpretation of the additivity will be the same (i.e., $\theta_{12}^{\prime}=0$ ), and only the magnitude of the nonadditivity (synergism or antagonism) will differ. Our model is, therefore, quite general, even though the data available to us did not allow for a validation of the marginal (i.e., single drug) concentration-response relationships it assumes.

To obtain a more reliable qualification and quantification of additivity, the concentrationresponse relationships of each drug alone should be identifiable using the experimental data. It has recently been demonstrated that the relationship between the increased total power of the electroencephalogram in rats treated by imipenem, an antibiotic with potential epileptogenic activity, followed the occurrence of seizures, and drug concentrations could be well described by a simplified Hill equation. ${ }^{20,21} \mathrm{~A}$ more recent study showed that the same holds true with norfloxacin. ${ }^{22}$ Not only the general shape of the concentration-effect relationship, but also the parameter values for each compound would be necessary to definitely characterize the theophylline-pefloxacin-norfloxacin convulsant interaction, but this information is not available at the moment. Although the data interpretation presented in this paper corresponds to the best that could be done with the current knowledge, one should keep in mind that it is only possible for us to refer to "apparent" additivity or "apparent" nonadditivity, without the possibility of a more precise interpretation.

Fundamentally, our PD interaction model unifies the analysis of direct and indirect interactions assays by linking an explicit effect threshold model to underlying dose-effect models for each drug studied. The combinations of any number of drugs can be used. Doses measured with limited precision (e.g., internal doses) can be used in input. The resulting uncertainty is fully and consistently accounted for by the statistical treatment of the data. The use of Bayesian statistical inference allows the inclusion of supplemental information on analytical assay precision, interindividual variability, or any other prior knowledge of the model parameters.

\section{ACKNOWLEDGMENTS}

We thank Pr. Eric Parent for helpful discussions. This work has been funded by the French Ministry of the Environment (BCRD AP 2001) and the U.S. National Science Foundation (SES-00-84368).

\section{APPENDIX A}

To extend the model presented in the Data Modeling section to a mixture with more than two drugs, a new concentration-response relationship should be defined. For a three-drug mixture, the standard polynomial model at the level of effect $y_{\mathrm{E}, j}$, for the experimental subject $j$, is given by:

$$
\begin{aligned}
y_{\mathrm{E}, j}= & \theta_{0}+\theta_{1} u_{1, j}+\theta_{2} u_{2, j}+\theta_{3} u_{3, j}+\theta_{12} u_{1, j} u_{2, j} \\
& +\theta_{13} u_{1, j} u_{3, j}+\theta_{23} u_{2, j} u_{3, j}+\theta_{123} u_{1, j} u_{2, j} u_{3, j}
\end{aligned}
$$

The simplest reparametrized surface (to fit to the data) can be written as,

$$
u_{1, j}=\frac{y_{\mathrm{E}, j}^{\prime}-\theta_{2}^{\prime} u_{2, j}-\theta_{3}^{\prime} u_{3, j}-\theta_{23}^{\prime} u_{2, j} u_{3, j}}{1+\theta_{12}^{\prime} u_{2, j}+\theta_{13}^{\prime} u_{3, j}+\theta_{123}^{\prime} u_{2, j} u_{3, j}}
$$

where

$$
y_{\mathrm{E}, j}^{\prime}=\frac{y_{\mathrm{E}, j}-\theta_{0}}{\theta_{1}}, \quad \theta_{k}^{\prime}=\frac{\theta_{k}}{\theta_{1}}
$$

The isobols corresponding to the two-two interactions can be obtained by setting one of the real 
concentration $u$ equal to zero. For a three-drug mixture, the set of parameterto estimate, $\Theta$, is

$$
\begin{aligned}
\Theta= & \left\{\theta_{2}^{\prime}, \theta_{3}^{\prime}, \theta_{12}^{\prime}, \theta_{13}^{\prime}, \theta_{23}^{\prime}, \theta_{123}^{\prime}, \overrightarrow{y_{\mathrm{E}}^{\prime}}, M_{y}^{\prime}, \sigma_{y}^{\prime},\right. \\
& \left.\overrightarrow{u_{1}}, \overrightarrow{u_{2}}, \overrightarrow{u_{3}}, \sigma_{1}, \sigma_{2}, \sigma_{3}\right\}
\end{aligned}
$$

\section{APPENDIX B}

The estimation of the parameters is performed in a Bayesian framework. In this appendix, the method for sampling the posterior probability $p\left(\Theta \mid x_{1}, x_{2}\right)$ is presented. To determine the distribution of $\Theta$ conditionally to $x_{1}$ and $x_{2}, p\left(\Theta \mid x_{1}, x_{2}\right)$, suppose that $x_{1}$ and $x_{2}$ are independent given $u_{1}$ and $u_{2}$. Applying Bayes' theorem gives

$$
\begin{aligned}
p\left(\Theta \mid x_{1}, x_{2}\right) \propto & p(\Theta) \prod_{j} p\left(x_{1, j} \mid u_{1, j}, \sigma_{1}\right) \\
& \times \prod_{j} p\left(x_{2, j} \mid u_{2, j}, \sigma_{2}\right)
\end{aligned}
$$

Conditional independence between $\overrightarrow{y_{\mathrm{E}}}$ and $\left(u_{1}\right.$, $u_{2}$ ), given the other components of $\Theta$, allows the following factorization:

$$
\begin{aligned}
p & \left(\Theta \mid x_{1}, x_{2}\right) \propto p\left(\theta, M_{y}, \sigma_{y}, \sigma_{1}, \sigma_{2}\right) \\
& \times p\left(\overrightarrow{y_{\mathrm{E}}} \mid M_{y}, \sigma_{y}\right) p\left(\overrightarrow{u_{1}}\left|\overrightarrow{u_{2}}\right| \overrightarrow{y_{\mathrm{E}}}, \theta\right) \\
& \times \prod_{j} p\left(x_{1, j} \mid u_{1, j}, \sigma_{1}\right) \prod_{j} p\left(x_{2, j} \mid u_{2, j}, \sigma_{2}\right)
\end{aligned}
$$

where $p\left(\Theta, M_{y}, \sigma_{y} \sigma_{1}, \sigma_{2}\right)$ is the prior distribution for the top-level model parameters, $p\left(\overrightarrow{y_{\mathrm{E}}} \mid M_{y}, \sigma_{y}\right)$ models interindividual variability, and $p\left(x_{1, j} \mid u_{1, j}, \sigma_{1}\right)$ and $p\left(x_{2, j} \mid u_{2, j}, \sigma_{2}\right)$ model measurement errors. The dose-response model is represented by $p\left(\overrightarrow{u_{1}}, \overrightarrow{u_{2}} \mid \overrightarrow{y_{\mathrm{E}}}, \theta\right)$.

We distinguish two cases: (i) the unit considered received a mixture of two agents, and (ii) the unit received only one agent. In the first case, $p\left(\overrightarrow{u_{1}}, \overrightarrow{u_{2}} \mid \overrightarrow{y_{\mathrm{E}}}, \theta\right)$ can be written as

$$
p\left(\overrightarrow{u_{1}}, \overrightarrow{u_{2}} \mid \overrightarrow{y_{\mathrm{E}}}, \theta\right)=p\left(\overrightarrow{u_{1}} \mid \overrightarrow{y_{\mathrm{E}}}, \theta\right) p\left(\overrightarrow{u_{2}} \mid \overrightarrow{u_{1}}, \overrightarrow{y_{\mathrm{E}}}, \theta\right)
$$

The isobol (eq. 4) is deterministic for $u_{2, j}$ conditioning on $u_{1, j}, y_{\mathrm{E}, j}$ and $\theta$, and $p\left(\overrightarrow{u_{2}} \mid \overrightarrow{u_{1}}, \overrightarrow{y_{\mathrm{E}}}, \theta\right)$ reduces to a strictly positive constant if (eq. 3) is verified, and to zero otherwise. So we obtain

$$
\begin{aligned}
p\left(\Theta \mid x_{1}, x_{2}\right) \propto & p\left(\theta, M_{y}, \sigma_{y}, \sigma_{1}, \sigma_{2}\right) \\
& \times \prod_{j} p\left(y_{\mathrm{E}, j} \mid M_{y}, \sigma_{y}\right) \prod_{j} p\left(u_{1, j} \mid y_{\mathrm{E}, j}, \theta\right) \\
& \times \prod_{j} p\left(x_{1, j} \mid u_{1, j}, \sigma_{1}\right) \prod_{j} p\left(x_{2, j} \mid u_{2, j}, \sigma_{2}\right)
\end{aligned}
$$

For units exposed to a single agent, the model is simpler. Consider an animal only exposed to agent A. The model's variables $\left(x_{2}\right.$ and $\left.u_{2}\right)$ corresponding to agent $\mathrm{B}$ disappear, and $p\left(\Theta \mid x_{1}, x_{2}\right)$ is

$$
\begin{aligned}
& p\left(\Theta \mid x_{1}, x_{2}\right) \propto p\left(\theta, M_{y}, \sigma_{y}, \sigma_{1}\right) p\left(\overrightarrow{y_{\mathrm{E}}} \mid M_{y}, \sigma_{y}\right) \\
& \times p\left(\overrightarrow{u_{1}} \mid \overrightarrow{y_{\mathrm{E}}}, \theta\right) \prod_{j} p\left(x_{1, j} \mid u_{1, j}, \sigma_{1}\right)
\end{aligned}
$$

and eqs. 4 and 5 (see text) give

$$
u_{1, j}=\frac{y_{\mathrm{E}, j}-\theta}{\theta_{1}}=y_{\mathrm{E}, j}^{\prime}
$$

Equation B.6 is deterministic for $u_{1, j}$ conditioning on $y_{\mathrm{E}, j}$ and $\theta$. So the term $p\left(\overrightarrow{u_{1}} \mid \overrightarrow{y_{\mathrm{E}}}, \theta\right)$ reduces to a constant if eq. 4 is verified and is zero otherwise. Replacing by the corresponding distributions, we have

$$
\begin{aligned}
p\left(\Theta \mid x_{1}, x_{2}\right) \propto & p\left(\theta, M_{y}, \sigma_{y}, \sigma_{1}\right) \times \prod_{j} p\left(y_{\mathrm{E}, j} \mid M_{y}, \sigma_{y}\right) \\
& \times \prod_{j} p\left(x_{1, j} \mid u_{1, j}, \sigma_{1}\right)
\end{aligned}
$$

\section{APPENDIX C}

This Appendix shows that the isobols of the $E_{\max }$ model can be characterized by eq. 5. For a twodrug mixture, the $E_{\max }$ model is given by

$$
y_{\mathrm{E}}=\frac{E_{\max }\left(\frac{x_{1}}{X_{1}}+\frac{x_{2}}{X_{2}}+\alpha \frac{x_{1}}{X_{1}} \frac{x_{2}}{X_{2}}\right)}{1+\left(\frac{x_{1}}{X_{1}}+\frac{x_{2}}{X_{2}}+\alpha \frac{x_{1}}{X_{1}} \frac{x_{2}}{X_{2}}\right)}
$$

where $y_{\mathrm{E}}$ is the response, $E_{\max }$ is the maximum response, $x_{i}$ is the concentration of drug $i, X_{i}$ is the drug $i$ concentration for which the effect is $E_{\max } / 2$, and $\alpha$ is the parameter describing the sign and strength of interactions between the two drugs. The corresponding isobol can be written as

$$
x_{2}=X_{2} \frac{\frac{y_{\mathrm{E}}}{E_{\max }-y_{\mathrm{E}}}-\frac{x_{1}}{X_{1}}}{1+\alpha \frac{x_{1}}{X_{1}}}
$$

Some parameters are not separately identifiable. The simplest reparametrized isobol is

$$
x_{2}=\frac{y_{\mathrm{E}}^{\prime}-x_{1}}{\theta^{\prime}+\alpha^{\prime} x_{1}}
$$

where

$$
y_{\mathrm{E}, j}^{\prime}=X_{1} \frac{y_{\mathrm{E}}}{E_{\max }-y_{\mathrm{E}}}, \quad \theta^{\prime}=\frac{X_{1}}{X_{2}}, \quad \alpha^{\prime}=\frac{\propto}{X_{2}}
$$


eq. C. 3 is then identical to the eq. 5 . We conclude that the expression of isobols is the same for the linear and the $\mathrm{E}_{\max }$ models.

\section{REFERENCES}

1. Delon A, Huguet F, Courtois P, Vierfond JM, Bouquet S, Couet W. 1997. Pharmacokinetic-pharmacodynamic contributions to the convulsant activity of pefloxacin and norfloxacin in rats. $\mathrm{J}$ Pharmacol Exp Ther 280:983-987.

2. Ramzan IM, Levy G. 1986. Kinetics of drug action in disease states. XVI. Pharmacodynamics of theophylline-induced seizures in rats. J Pharmacol Exp Ther 236:708-713.

3. Levasseur LM, Delon A, Greco WR, Faury P, Bouquet S, Couet W. 1998. Development of a new quantitative approach for the isobolographic assessment of the convulsant interaction between pefloxacin and theophylline in rats. Pharm Res 15: 1069-1076.

4. Delon A, Levasseur LM, Giraudon M, Bouquet S, Couet W. 1999. Antagonistic interaction between the convulsant activities of pefloxacin and its main metabolite norfloxacin in rats. Pharm Res 16:18941897.

5. Cadart M, Marchand S, Pariat C, Bouquet S, Couet W. 2002. Ignoring pharmacokinetics may lead to isoboles misinterpretation: Illustration with the norfloxacin- theophylline convulsant interaction in rats. Pharm Res 19:209-214.

6. Delon A, Bouquet S, Huguet F, Brunet V, Courtois P, Couet W. 1999. Pharmacokinetic-pharmacodynamic contributions to the convulsant activity of fluoroquinolones in rats. Antimicrob Agents Chemother 43:1511-1515.

7. Carter WH, Jr. 1995. Relating isobolograms to response surfaces. Toxicology 105:181-188.

8. Gennings C, Carter WH, Jr., Campbell ED, Staniswalis JG, Martin TJ, Martin BR, White KL, Jr. 1990. Isobolographic characterization of drug interactions incorporating biological variability. J Pharmacol Exp Ther 252:208-217.

9. Sheiner LB, Ludden TM. 1992. Population pharmacokinetics/dynamics. Annual Review of Pharmacology and Toxicology 32:185-209.
10. Finney DJ. 1952. Probit analysis, second edition. Cambridge: Cambridge University Press, 350 p.

11. Pearl J, Geiger D, Verma T. 1990. The logic of influence diagrams. In: Oliver RM, Smith JQ, editors. Influence diagrams, belief nets and decision analysis. Chichester: Wiley Series in Probability and Mathematical Statistics, pp 67-87.

12. Bernardo JM, Smith AFM. 1994. Bayesian theory. New York: Wiley, 586 p.

13. Gelman A, Carlin JB, Stern HS, Rubin DB. 1995. Bayesian data analysis. London: Chapman \& Hall, $526 \mathrm{p}$.

14. Gilks WR, Richardson S, Spiegelhalter DJ. 1996. Markov chain Monte Carlo in practice. London: Chapman \& Hall; $486 \mathrm{p}$.

15. Bois FY. Maszle D. 1997. MCSim: A simulation program. J Stat Software 2(9): http://www.stat. ucla.edu/journals/jss/v02/i09.

16. Derendorf H, Meibohm B. 1999. Modeling of pharmacokinetic/pharmacodynamic (PK/PD) relationships: Concepts and perspectives. Pharm Res 16:176-185.

17. Seber GAF, Wild CJ. 1989. Nonlinear regression. New York: John Wiley \& Sons, 768 p.

18. Bennett J, Wakefield J. 2001. Errors-in-variables in joint population pharmacokinetic/pharmacodynamic modeling. Biometrics 57:803-812.

19. Dexter F. 1995. Statistical analysis of drug interactions in anesthesia. J Theor Biol 172:305314.

20. Dupuis A, Couet W, Paquereau J, Debarre S, Portron A, Jamois C, Bouquet S. 2001. Pharmacokinetic-pharmacodynamic modeling of the electroencephalogram effect of imipenem in healthy rats. Antimicrob Agents Chemother 45:16821687.

21. Dupuis A, Limosin A, Paquereau J, Mimoz O, Couet W, Bouquet S. 2001. Pharmacokineticpharmacodynamic modeling of electroencephalogram effect of imipenem in rats with acute renal failure. Antimicrob Agents Chemother 45:36073609.

22. Chenel M, Barbot A, Dupuis A, Mimoz O, Paquereau J, Bouquet S, Couet W. 2003. Pharmacokineticpharmacodynamic modeling of electroencephalogram effect of norfloxacin in rats. Antimicrob Agents Chemother 47:1952-1957. 